\title{
Casimir-Polder interaction of fullerene molecules with surfaces
}

\author{
Stefan Yoshi Buhmann and Stefan Scheel \\ Quantum Optics and Laser Science, Blackett Laboratory, Imperial College London, \\ Prince Consort Road, London SW7 2BW, United Kingdom
}

\begin{abstract}
Simen A. Ellingsen
Department of Energy and Process Engineering, Norwegian University of Science and Technology, N-7491 Trondheim, Norway

Klaus Hornberger and Andreas Jacob

Unversität Duisburg-Essen, Fakultät für Physik, Lotharstraße 1-21, 47057 Duisburg, Germany
\end{abstract}

(Dated: November 5, 2018)

\begin{abstract}
We calculate the thermal Casimir-Polder potential of $\mathrm{C}_{60}$ and $\mathrm{C}_{70}$ fullerene molecules near gold and silicon nitride surfaces, motivated by their relevance for molecular matter wave interference experiments. We obtain the coefficients governing the asymptotic power laws of the interaction in the thermal, retarded and nonretarded distance regimes and evaluate the full potential numerically. The interaction is found to be dominated by electronic transitions, and hence independent of the internal temperature of the molecules. The contributions from phonon transitions, which are affected by the molecular temperature, give rise to only a small correction. Moreover, we find that the sizeable molecular line widths of thermal fullerenes may modify the nonretarded interaction, depending on the model used. Detailed measurements of the nonretarded potential of fullerene thus allow one to distinguish between different theories of incorporating damping.
\end{abstract}

PACS numbers: $31.30 . j \mathrm{~h}, 12.20 .-\mathrm{m}, 34.35 .+\mathrm{a}, 42.50 . \mathrm{Nn}$

\section{INTRODUCTION}

It is a remarkable feature of fullerene buckyballs that they can exist as delocalized quantum waves [1], as is proven almost routinely in matter wave interference experiments [2]. Such interferometer setups involve nanomechanical grating structures, typically made of gold or silicon nitride. As the molecules pass through the grating slits they experience an attractive dispersion force between the polarisable molecule and the grating wall. Even though this Casimir-Polder (CP) interaction is weak, it must be accounted for in predictions of the interference fringes $[3,4]$.

The influence of dispersion forces is particularly strong in modern near-field interference setups where many different interference orders contribute resonantly, implying that even tiny distortions of the molecular wave fronts affect the fringe pattern [4]. In these experiments, it is the presence of the Casimir-Polder interaction which impedes the demonstration of interference with even larger and more polarisable particles [5]. At the same time, this strong sensitivity of the fullerene matter waves provides a means of verifying the precise value and functional form of the dispersion forces. It is therefore important to have a reliable description of the expected Casimir-Polder potential available, which should also account for molecules not in thermal equilibrium with their environment, given that the beam is usually produced by thermal sublimation.

Casimir-Polder interactions have been studied intensively in recent years, though mainly focused on atoms [6]. Such studies include thermal equilibrium [7] as well as non-equilibrium situations [8, 9] in which the inter- nal temperature of the microscopic (atomic or molecular) system can be vastly different from that of the macroscopic environment. Carbon-based nanostructures have been of particular interest due to applications. In this context, the interaction of a carbon nanotube with a surface has been studied [10].

Motivated by the mentioned matter-wave interference experiments we now discuss and evaluate in detail the $\mathrm{CP}$ interaction of $\mathrm{C}_{60}$ and $\mathrm{C}_{70}$ fullerenes with planar surfaces made of gold or silicon nitride $\left(\mathrm{SiN}_{x}\right.$ or $\left.\mathrm{Si}_{3} \mathrm{~N}_{4}\right)$. In Sect. III, we summarise the basic equations that govern the CP potential, determine the molecular polarisabilities of the fullerenes from spectroscopic data, and list the material parameters of the surface materials. In Sect.III, we calculate the CP potentials by both numerical and analytical means and discuss our results. A short summary is given in Sect. IV]

\section{BASIC EQUATIONS AND PARAMETERS}

We begin by presenting the theory of the thermal CP potential and by recording the molecular and material properties as obtained from optical data.

\section{A. Thermal Casimir-Polder potential}

We consider a non-magnetic, isotropic molecule of internal temperature $T_{\mathrm{m}}$ placed at a distance $z$ from a plane non-magnetic surface of permittivity $\varepsilon(\omega)$, with both surface and environment being held at uniform temperature T. As shown in Refs. 9] and [11], the thermal CP poten- 
tial of the molecule can be given as a sum of non-resonant and resonant contributions,

$$
U(z)=U_{\text {nres }}(z)+U_{\text {res }}(z)
$$

The non-resonant contribution is due to virtual photons and it is given by a sum

$$
\begin{aligned}
U_{\mathrm{nres}}(z)= & \frac{\mu_{0} k_{\mathrm{B}} T}{8 \pi} \sum_{j=0}^{\infty}\left[\alpha_{T_{\mathrm{m}}}\left(\mathrm{i} \xi_{j}\right)+\alpha_{T_{\mathrm{m}}}\left(-\mathrm{i} \xi_{j}\right)\right] \\
& \times \int_{\xi_{j} / c}^{\infty} \mathrm{d} \kappa^{\perp} \mathrm{e}^{-2 \kappa^{\perp} z}\left[\xi_{j}^{2} r_{s}\left(\xi_{j}, \kappa^{\perp}\right)\right. \\
& \left.-\left(2 \kappa^{\perp 2} c^{2}-\xi_{j}^{2}\right) r_{p}\left(\xi_{j}, \kappa^{\perp}\right)\right]
\end{aligned}
$$

over the purely imaginary Matsubara frequencies $\mathrm{i} \xi_{j}$ with $\xi_{j}=\left(2 \pi k_{\mathrm{B}} T / \hbar\right) j$, where the prime indicates that the $j=0$ term carries half-weight. The properties of the molecule are represented by its thermal polarisability

$$
\alpha_{T_{\mathrm{m}}}(\omega)=\sum_{n} p_{n}\left(T_{\mathrm{m}}\right) \alpha_{n}(\omega)
$$

where

$$
p_{n}\left(T_{\mathrm{m}}\right)=\frac{\mathrm{e}^{-E_{n} /\left(k_{\mathrm{B}} T_{\mathrm{m}}\right)}}{\sum_{k} \mathrm{e}^{-E_{k} /\left(k_{\mathrm{B}} T_{\mathrm{m}}\right)}}
$$

denotes the populations of the molecular eigenstates with energies $E_{n}$ and

$$
\alpha_{n}(\omega)=\lim _{\epsilon \rightarrow 0_{+}} \frac{2}{3 \hbar} \sum_{k} \frac{\omega_{k n}\left|\boldsymbol{d}_{n k}\right|^{2}}{\omega_{k n}^{2}-\omega^{2}-\mathrm{i} \omega\left(\Gamma_{n}+\Gamma_{k}\right) / 2}
$$

$\left[\omega_{k n}=\left(E_{k}-E_{n}\right) / \hbar\right.$ : molecular transition frequencies, $\boldsymbol{d}_{n k}$ : electric dipole matrix elements, $\Gamma_{n}$ : level widths/damping constants, $\Gamma_{0}=0$ ] are the associated polarisabilities. The material properties of the surface enter via the reflection coefficients for $s$ - and $p$-polarised waves

$$
r_{s}\left(\xi, \kappa^{\perp}\right)=\frac{\kappa^{\perp}-\kappa_{1}^{\perp}}{\kappa^{\perp}+\kappa_{1}^{\perp}}, \quad r_{p}\left(\xi, \kappa^{\perp}\right)=\frac{\varepsilon(\mathrm{i} \xi) \kappa^{\perp}-\kappa_{1}^{\perp}}{\varepsilon(\mathrm{i} \xi) \kappa^{\perp}+\kappa_{1}^{\perp}}
$$

with $\kappa_{1}^{\perp}=\sqrt{\kappa^{\perp 2}+[\varepsilon(\mathrm{i} \xi)-1] \xi^{2} / c^{2}}$.

The resonant contribution to the potential is due to the absorption and stimulated emission of real photons, it reads

$$
\begin{aligned}
& U_{\text {res }}(z) \\
& =\frac{\mu_{0}}{12 \pi} \sum_{n} p_{n}\left(T_{\mathrm{m}}\right)\left\{\sum_{k<n}\left[n_{T}\left(\omega_{n k}\right)+1\right]-\sum_{k>n} n_{T}\left(\omega_{k n}\right)\right\} \\
& \quad \times \omega_{n k}^{2}\left|\boldsymbol{d}_{n k}\right|^{2} \int_{0}^{\infty} \mathrm{d} k^{\|} \frac{k^{\|}}{k^{\perp}}\left\{\operatorname{Im}\left[\mathrm{e}^{2 \mathrm{i} k^{\perp} z} r_{s}\left(\left|\omega_{k n}\right|, k^{\|}\right)\right]\right. \\
& \left.\quad-\left(2 \frac{k^{\perp 2} c^{2}}{\omega_{n k}^{2}}-1\right) \operatorname{Im}\left(\mathrm{e}^{2 \mathrm{i} k^{\perp} z} r_{p}\left(\left|\omega_{k n}\right|, k^{\|}\right)\right]\right\}
\end{aligned}
$$

with

$$
n_{T}(\omega)=\frac{1}{\mathrm{e}^{\hbar \omega /\left(k_{\mathrm{B}} T\right)}-1}
$$

denoting the thermal photon number. The reflection coefficients for real frequencies can be given as

$$
r_{s}\left(\omega, k^{\|}\right)=\frac{k^{\perp}-k_{1}^{\perp}}{k^{\perp}+k_{1}^{\perp}}, \quad r_{p}\left(\omega, k^{\|}\right)=\frac{\varepsilon(\omega) k^{\perp}-k_{1}^{\perp}}{\varepsilon(\omega) k^{\perp}+k_{1}^{\perp}}
$$

with $k^{\perp}=\sqrt{\omega^{2} / c^{2}-k^{\| 2}}$ if $k^{\|} \leq \omega / c, k^{\perp}=\mathrm{i} \sqrt{k^{\| 2}-\omega^{2} / c^{2}}$ if $k^{\|} \geq \omega / c$ and $k_{1}^{\perp}=\sqrt{\varepsilon(\omega) \omega^{2} / c^{2}-k^{\| 2}}$ with $\operatorname{Im} k_{1}^{\perp}>0$.

We stress that the molecule is out of thermal equilibrium with its environment whenever $T_{\mathrm{m}} \neq T$. Here, the thermal polarisability as well as the internal-state populations of the molecule depend on its internal temperature $T_{\mathrm{m}}$, while the Matsubara frequencies and thermal photon numbers are given in terms of the environment temperature $T$.

\section{B. Molecular and material properties}

The dielectric permittivity $\varepsilon(\omega)$ of thin fullerene films in the optical regime has been measured by means of electron energy-loss spectroscopy [12] as well as in the gas phase [13]. The frequencies and dipole matrix elements of the corresponding electronic transitions of single fullerene molecules can be deduced from the data in Ref. [12] in a two-step procedure.

We first apply a simultaneous least-squares fit of an $n$-oscillator model

$$
\varepsilon(\omega)=\varepsilon_{\infty}+\sum_{i=1}^{n} \frac{f_{i} \Omega_{i}^{2}}{\Omega_{i}^{2}-\omega^{2}-\mathrm{i} \gamma_{i} \omega}
$$

to the measured data for the real and imaginary parts of the permittivity as taken from Ref. [12]. The fits with $n=9$ oscillators for $\mathrm{C}_{60}$ and $n=7$ for $\mathrm{C}_{70}$ are illustrated in Figs. 1 and 2. The obtained fit parameters $\Omega_{i}, f_{i}$ and $\Gamma_{i}$ are given in Tabs. I and II]

Next, we relate the fitted permittivity to the molecular polarisability by means of the Clausius-Mosotti law

$$
\alpha(\omega)=\frac{3 \varepsilon_{0}}{\eta} \frac{\varepsilon(\omega)-1}{\varepsilon(\omega)+2}
$$

where $\eta$ is the number density of fullerene molecules in the film. For a face-centred-cubic crystal structure of the fullerene molecules in the thin film, one has $\eta=4 / a^{3}$ with a lattice constants $a=1.42 \times 10^{-9} \mathrm{~m}$ for $\mathrm{C}_{60}$ and $a=1.51 \times 10^{-9} \mathrm{~m}$ for $\mathrm{C}_{70}[12$. We employ a decomposition of the resulting expression into partial fractions to write it in the form

$$
\alpha_{0}(\omega)=\frac{2}{3 \hbar} \sum_{k} \frac{\omega_{k 0}\left|\boldsymbol{d}_{0 k}\right|^{2}}{\omega_{k 0}^{2}-\omega^{2}-\mathrm{i} \omega \Gamma_{k} / 2},
$$



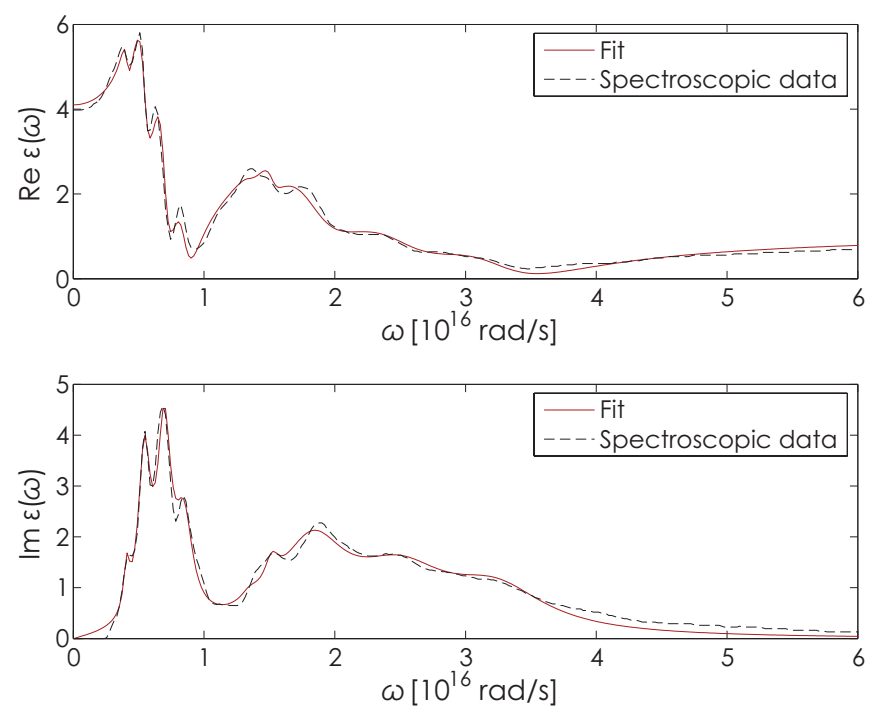

FIG. 1: 9-oscillator fit (solid line) to the spectroscopic data (dashed line) of the permittivity of a thin $\mathrm{C}_{60}$ film.
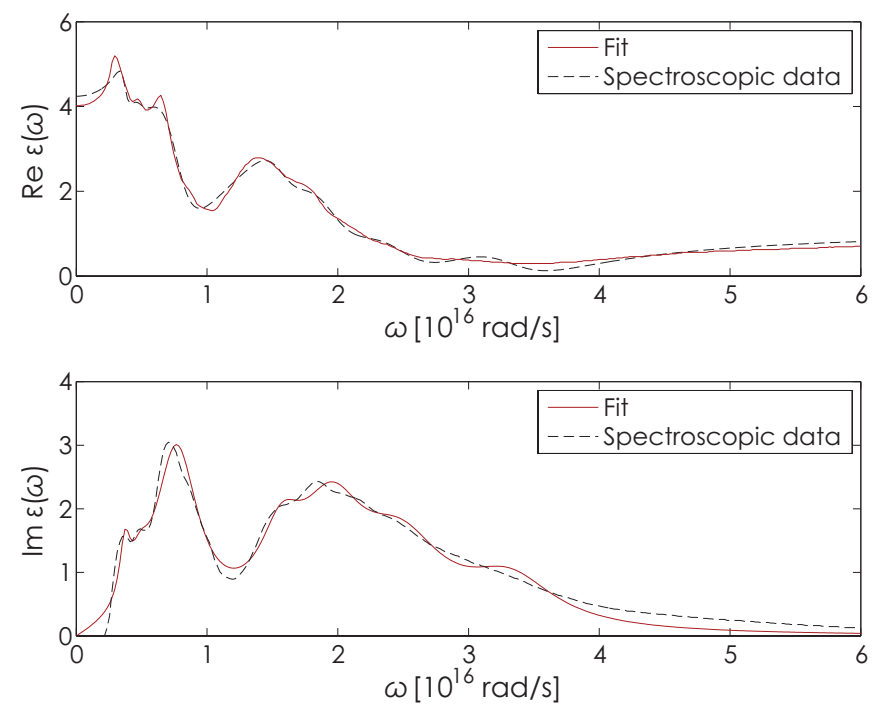

FIG. 2: 7-oscillator fit (solid line) to the spectroscopic data (dashed line) of the permittivity of a thin $\mathrm{C}_{70}$ film.

from which the transition frequencies $\omega_{k 0}$, dipole matrix elements $d_{0 k}$ and excited-state widths $\Gamma_{k}$ can be read off. They are given in Tabs. III and IV] Note that we have used the ground-state polarisability $\alpha_{0}(\omega)$ rather than its thermal counterpart (2.3). This is a good approximation for the considered electronic transitions whose frequencies are much higher than the thermal frequency $k_{\mathrm{B}} T_{\mathrm{m}} / \hbar=3.93 \times 10^{13} \mathrm{rad} / \mathrm{s}$ corresponding to the temperature of the molecules in the experiment [12], $T_{\mathrm{m}}=300 \mathrm{~K}$.

In addition to the optical transitions, four phonon transitions have been identified for $\mathrm{C}_{60}$ in the infrared

\begin{tabular}{|c|c|c|}
\hline$\Omega_{i}[\mathrm{rad} / \mathrm{s}]$ & $f_{i}$ & $\gamma_{i}[\mathrm{rad} / \mathrm{s}]$ \\
\hline $4.10 \times 10^{15}$ & 0.120 & $5.44 \times 10^{14}$ \\
$5.47 \times 10^{15}$ & 0.663 & $1.14 \times 10^{15}$ \\
$6.99 \times 10^{15}$ & 0.664 & $1.28 \times 10^{15}$ \\
$8.51 \times 10^{15}$ & 0.348 & $1.60 \times 10^{15}$ \\
$1.35 \times 10^{16}$ & 0.0270 & $1.72 \times 10^{15}$ \\
$1.52 \times 10^{16}$ & 0.0471 & $1.33 \times 10^{15}$ \\
$1.85 \times 10^{16}$ & 0.554 & $6.29 \times 10^{15}$ \\
$2.54 \times 10^{16}$ & 0.403 & $9.28 \times 10^{15}$ \\
$3.27 \times 10^{16}$ & 0.229 & $9.31 \times 10^{15}$ \\
\hline
\end{tabular}

TABLE I: Fit parameters obtained for the permittivity of $\mathrm{C}_{60}$ films in the optical regime. $\varepsilon_{\infty}=1.0463$

\begin{tabular}{|c|c|c|}
\hline$\Omega_{i}[\mathrm{rad} / \mathrm{s}]$ & $f_{i}$ & $\gamma_{i}[\mathrm{rad} / \mathrm{s}]$ \\
\hline $3.76 \times 10^{15}$ & 0.245 & $9.91 \times 10^{14}$ \\
$4.97 \times 10^{15}$ & 0.170 & $1.70 \times 10^{15}$ \\
$7.92 \times 10^{15}$ & 1.39 & $4.05 \times 10^{15}$ \\
$1.58 \times 10^{16}$ & 0.276 & $4.05 \times 10^{15}$ \\
$1.98 \times 10^{16}$ & 0.502 & $6.17 \times 10^{15}$ \\
$2.51 \times 10^{16}$ & 0.393 & $8.19 \times 10^{15}$ \\
$3.34 \times 10^{16}$ & 0.186 & $8.45 \times 10^{15}$ \\
\hline
\end{tabular}

TABLE II: Fit parameters obtained for the permittivity of $\mathrm{C}_{70}$ films in the optical regime. $\varepsilon_{\infty}=1.0827$

frequency regime. The respective permittivity data obtained from Fourier transform infrared experiments have been fitted to a model of the kind given by Eq. (2.10) [14], with the parameters being listed in Tab. V] To obtain the respective molecular polarisability, we make use of the Clausius-Mosotti law (2.11). As the measurements have been performed at room temperature $\left(T_{\mathrm{m}}=300 \mathrm{~K}\right)$ where the phonons are excited to a considerable degree, we have to employ the thermal polarisability (2.3). According to Eqs. (2.4) and (2.5), the latter can be written in the form

$$
\begin{aligned}
& \alpha_{T_{\mathrm{m}}}(\omega)= \frac{2}{3 \hbar} \sum_{n, k} p_{n}\left(T_{\mathrm{m}}\right) \frac{\omega_{k n}\left|\boldsymbol{d}_{n k}\right|^{2}}{\omega_{k n}^{2}-\omega^{2}-\mathrm{i} \omega\left(\Gamma_{n}+\Gamma_{k}\right) / 2} \\
&=\frac{2}{3 \hbar} \sum_{n<k} p_{n k}\left(T_{\mathrm{m}}\right) \tanh \left(\frac{\hbar \omega_{k n}}{2 k_{\mathrm{B}} T_{\mathrm{m}}}\right) \\
& \times \frac{\omega_{k n}\left|\boldsymbol{d}_{n k}\right|^{2}}{\omega_{k n}^{2}-\omega^{2}-\mathrm{i} \omega\left(\Gamma_{n}+\Gamma_{k}\right) / 2}
\end{aligned}
$$

with $p_{n k}(T)=p_{n}(T)+p_{k}(T)$. Assuming that all observed phonon transitions are from the ground state $(n=0)$, we 


\begin{tabular}{|c|c|c|}
\hline$\omega_{k 0}[\mathrm{rad} / \mathrm{s}]$ & $d_{0 k}[\mathrm{Cm}]$ & $\Gamma_{k}[\mathrm{rad} / \mathrm{s}]$ \\
\hline $4.14 \times 10^{15}$ & $7.93 \times 10^{-30}$ & $1.10 \times 10^{15}$ \\
$5.73 \times 10^{15}$ & $2.36 \times 10^{-29}$ & $2.32 \times 10^{15}$ \\
$7.43 \times 10^{15}$ & $3.58 \times 10^{-29}$ & $2.65 \times 10^{15}$ \\
$8.97 \times 10^{15}$ & $4.93 \times 10^{-29}$ & $3.23 \times 10^{15}$ \\
$1.36 \times 10^{16}$ & $1.09 \times 10^{-29}$ & $3.47 \times 10^{15}$ \\
$1.53 \times 10^{16}$ & $1.65 \times 10^{-29}$ & $2.73 \times 10^{15}$ \\
$1.98 \times 10^{16}$ & $7.28 \times 10^{-29}$ & $1.28 \times 10^{16}$ \\
$2.70 \times 10^{16}$ & $9.42 \times 10^{-29}$ & $1.83 \times 10^{16}$ \\
$3.43 \times 10^{16}$ & $1.11 \times 10^{-28}$ & $1.85 \times 10^{16}$ \\
\hline
\end{tabular}

TABLE III: Transition frequencies, dipole matrix elements and widths of the electronic transitions of $\mathrm{C}_{60}$.

\begin{tabular}{|c|c|c|}
\hline$\omega_{k 0}[\mathrm{rad} / \mathrm{s}]$ & $d_{0 k}[\mathrm{Cm}]$ & $\Gamma_{k}[\mathrm{rad} / \mathrm{s}]$ \\
\hline $3.83 \times 10^{15}$ & $1.40 \times 10^{-29}$ & $2.01 \times 10^{15}$ \\
$5.03 \times 10^{15}$ & $1.50 \times 10^{-29}$ & $3.43 \times 10^{15}$ \\
$9.04 \times 10^{15}$ & $6.91 \times 10^{-29}$ & $8.12 \times 10^{15}$ \\
$1.63 \times 10^{16}$ & $4.03 \times 10^{-29}$ & $8.28 \times 10^{15}$ \\
$2.10 \times 10^{16}$ & $7.90 \times 10^{-29}$ & $1.25 \times 10^{16}$ \\
$2.69 \times 10^{16}$ & $1.15 \times 10^{-28}$ & $1.60 \times 10^{16}$ \\
$3.48 \times 10^{16}$ & $1.13 \times 10^{-28}$ & $1.68 \times 10^{16}$ \\
\hline
\end{tabular}

TABLE IV: Transition frequencies, dipole matrix elements and widths of the electronic transitions of $\mathrm{C}_{70}$.

have

$$
\begin{aligned}
& \alpha_{T_{\mathrm{m}}}(\omega)=\frac{2}{3 \hbar} \sum_{k} p_{0 k}\left(T_{\mathrm{m}}\right) \tanh \left(\frac{\hbar \omega_{k 0}}{2 k_{\mathrm{B}} T_{\mathrm{m}}}\right) \\
& \times \frac{\omega_{k 0}\left|\boldsymbol{d}_{0 k}\right|^{2}}{\omega_{k 0}^{2}-\omega^{2}-\mathrm{i} \omega \Gamma_{k} / 2} .
\end{aligned}
$$

The transition frequencies and dipole matrix elements can then be readily obtained by comparing with the fit for Eq. (2.11), with the results being given in Tab. VI

We are going to study the interaction of fullerene molecules with $\mathrm{Au}, \mathrm{SiN}_{x}$ and $\mathrm{Si}_{3} \mathrm{~N}_{4}$. The permittivity of $\mathrm{Au}$ can be given as

$$
\varepsilon(\omega)=1-\frac{\Omega_{0}^{2}}{\omega\left(\omega+\mathrm{i} \gamma_{0}\right)}+\sum_{i=1}^{6} \frac{f_{i} \Omega_{i}^{2}}{\Omega_{i}^{2}-\omega^{2}-\mathrm{i} \gamma_{i} \omega}
$$

where the first term is the response of the conduction electrons as described by a Drude model [15] and the Lorentz-type contributions are due to atomic transitions (6-oscillator fit [16] based on data from Refs. 17] and [18]). Values for the model parameters as taken from the above mentioned references are listed in Tab. VII.

Diffraction experiments frequently use gratings made of low-pressure chemical vapour deposited silicon nitride. The imaginary part of the permittivity of this (nonstoichiometric) $\mathrm{SiN}_{x}$ has been determined from optical

\begin{tabular}{|c|c|c|}
\hline$\Omega_{i}[\mathrm{rad} / \mathrm{s}]$ & $f_{i}$ & $\gamma_{i}[\mathrm{rad} / \mathrm{s}]$ \\
\hline $9.91 \times 10^{13}$ & 0.024 & $4.33 \times 10^{11}$ \\
$1.08 \times 10^{14}$ & 0.007 & $6.03 \times 10^{11}$ \\
$2.23 \times 10^{14}$ & 0.0011 & $5.46 \times 10^{11}$ \\
$2.69 \times 10^{14}$ & 0.001 & $6.40 \times 10^{11}$ \\
\hline
\end{tabular}

TABLE V: Fit parameters for the permittivity of $\mathrm{C}_{60}$ films in the infrared regime.

\begin{tabular}{|c|c|c|}
\hline$\omega_{k 0}[\mathrm{rad} / \mathrm{s}]$ & $d_{0 k}[\mathrm{Cm}]$ & $\Gamma_{k}[\mathrm{rad} / \mathrm{s}]$ \\
\hline $9.95 \times 10^{13}$ & $1.69 \times 10^{-30}$ & $8.67 \times 10^{11}$ \\
$1.09 \times 10^{14}$ & $1.00 \times 10^{-30}$ & $1.21 \times 10^{12}$ \\
$2.23 \times 10^{14}$ & $5.33 \times 10^{-31}$ & $1.09 \times 10^{12}$ \\
$2.69 \times 10^{14}$ & $5.58 \times 10^{-31}$ & $1.28 \times 10^{12}$ \\
\hline
\end{tabular}

TABLE VI: Transition frequencies, dipole matrix elements and widths of the phonon transitions of $\mathrm{C}_{60}$.

measurements. As reported in Ref. [19], one has

$$
\operatorname{Im} \varepsilon(\omega)=\Theta\left(\omega-\Omega_{T}\right) \frac{f \Omega \gamma\left(\omega-\Omega_{T}\right)^{2}}{\left[\left(\omega^{2}-\Omega^{2}\right)^{2}-\gamma^{2} \omega^{2}\right] \omega}
$$

with parameters $\Omega_{T}=3.48 \times 10^{15} \mathrm{rad} / \mathrm{s}, \Omega=1.09 \times$ $10^{16} \mathrm{rad} / \mathrm{s}, f=1.13 \times 10^{17} \mathrm{rad} / \mathrm{s}$ and $\gamma=1.16 \times 10^{16} \mathrm{rad} / \mathrm{s}$. The permittivity at imaginary frequencies as required for the non-resonant CP potential can be obtained from the Kramers-Kronig relation

$$
\varepsilon(\mathrm{i} \xi)=\frac{2}{\pi} \int_{0}^{\infty} \mathrm{d} \omega \frac{\omega \operatorname{Im} \varepsilon(\omega)}{\omega^{2}+\xi^{2}} .
$$

In particular, $\varepsilon(0)=3.87$.

Alternatively, we also consider non-crystalline $\mathrm{Si}_{3} \mathrm{~N}_{4}$. The real and imaginary parts of its permittivity have been reported over a wide frequency range [20]. We have fitted this data with a single-resonance 4-parameter semiquantum model [21],

$$
\varepsilon(\omega)=\frac{\Omega_{\mathrm{L}}^{2}-\omega^{2}-\mathrm{i} \omega \gamma_{\mathrm{L}}}{\Omega_{\mathrm{T}}^{2}-\omega^{2}-\mathrm{i} \omega \gamma_{\mathrm{T}}} .
$$

The fit, as displayed in Fig. 3, yields the parameters $\Omega_{L}=$ $2.69 \times 10^{16} \mathrm{rad} / \mathrm{s}, \Omega_{T}=1.33 \times 10^{16} \mathrm{rad} / \mathrm{s}, \gamma_{L}=3.05 \times$ $10^{16} \mathrm{rad} / \mathrm{s}$, and $\gamma_{T}=6.40 \times 10^{15} \mathrm{rad} / \mathrm{s}$. This yields a static permittivity $\varepsilon(0)=4.10$.

\section{CASIMIR-POLDER POTENTIAL OF FULLERENE}

Using the basic formulas from Sect. IA together with the molecular and material parameters from Sec. IIB we can now evaluate the $\mathrm{CP}$ potential. We begin by calculating the $\mathrm{CP}$ potential associated with electronic transitions. As seen from Tabs. III and IV the electronic transition frequencies of $\mathrm{C}_{60}$ and $\mathrm{C}_{70}$ are much larger than the 


\begin{tabular}{|c|c|c|c|}
\hline$i$ & $\Omega_{i}[\mathrm{rad} / \mathrm{s}]$ & $f_{i}$ & $\gamma_{i}[\mathrm{rad} / \mathrm{s}]$ \\
\hline 0 & $1.37 \times 10^{16}$ & & $5.32 \times 10^{13}$ \\
1 & $4.63 \times 10^{15}$ & 0.762 & $1.14 \times 10^{15}$ \\
2 & $6.30 \times 10^{15}$ & 2.41 & $2.81 \times 10^{15}$ \\
3 & $8.20 \times 10^{15}$ & 0.0926 & $1.52 \times 10^{15}$ \\
4 & $1.29 \times 10^{16}$ & 2.14 & $1.06 \times 10^{16}$ \\
5 & $2.05 \times 10^{16}$ & 0.244 & $9.12 \times 10^{15}$ \\
6 & $3.27 \times 10^{16}$ & 0.670 & $1.37 \times 10^{16}$ \\
\hline
\end{tabular}

TABLE VII: Model parameters for the permittivity of $\mathrm{Au}$.
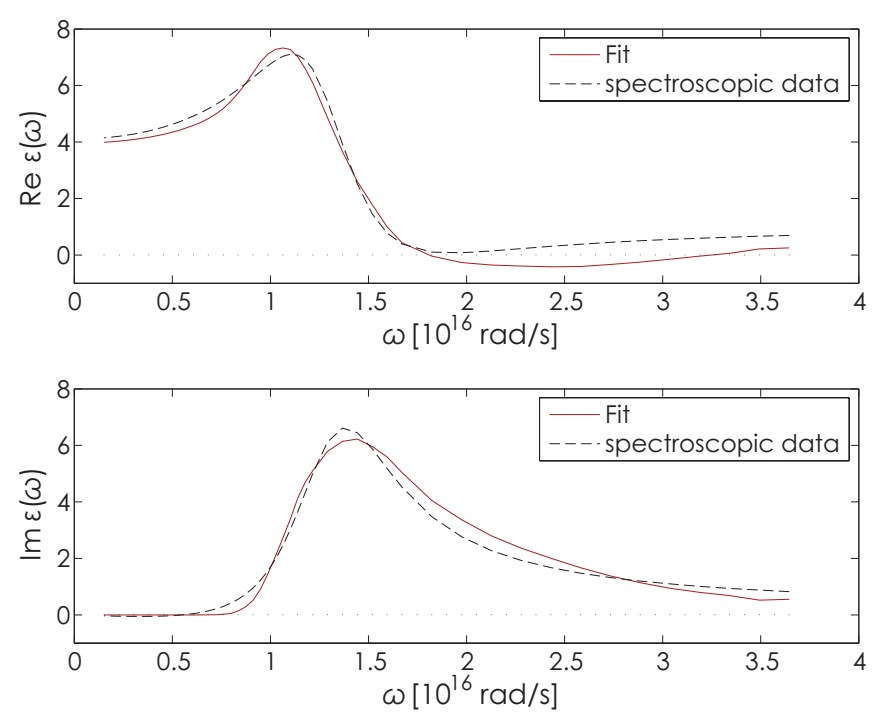

FIG. 3: 4-parameter semi-quantum model fit (solid line) to the spectroscopic data (dashed line) of the permittivity $\mathrm{Si}_{3} \mathrm{~N}_{4}$.

respective thermal frequency $k_{\mathrm{B}} T / \hbar=3.93 \times 10^{13} \mathrm{rad} / \mathrm{s}$ even at room temperature. As a consequence, the thermal photon numbers $n_{T}\left(\omega_{n k}\right)$ are extremely small. The molecules have shown interference at internal temperatures of $2500 \mathrm{~K}$ [23, 24] , and they are stable up to $6000 \mathrm{~K}$ [22]. Even at the latter temperature, the thermal frequency $k_{\mathrm{B}} T_{\mathrm{m}} / \hbar=7.86 \times 10^{14} \mathrm{rad} / \mathrm{s}$ is much smaller than the molecular transition frequencies, so the molecule is essentially in its electronic ground state, $p_{n}\left(T_{\mathrm{m}}\right)=\delta_{n 0}$. As a result, the resonant CP potential (2.7) vanishes and the $\mathrm{CP}$ potential (2.1) is entirely given by the nonresonant contribution (2.2) which simplifies to

$$
\begin{aligned}
& U(z)=\frac{\mu_{0} k_{\mathrm{B}} T}{8 \pi} \sum_{j=0}^{\infty} \xi_{j}^{2}\left[\alpha_{0}\left(\mathrm{i} \xi_{j}\right)+\alpha_{0}\left(-\mathrm{i} \xi_{j}\right)\right] \\
& \times \int_{\xi_{j} / c}^{\infty} \mathrm{d} \kappa^{\perp} \mathrm{e}^{-2 \kappa^{\perp} z}\left[r_{s}-\left(2 \frac{\kappa^{\perp 2} c^{2}}{\xi_{j}^{2}}-1\right) r_{p}\right] .
\end{aligned}
$$

As first shown in Ref. 25], we may distinguish three asymptotic regimes where the potential reduces to simple power laws. At distances much larger than the wavelength of the predominant thermal photons, $z \gg$ $\hbar c /\left(k_{\mathrm{B}} T\right)$, the Matsubara sum is dominated by its first term. Using Eq. (2.6) and carrying out the $\kappa^{\perp}$-integral, we have

$$
U(z)=-\frac{C_{3 T}}{z^{3}}
$$

with $\left[\alpha_{0}(0) \equiv \alpha_{0}\right]$

$$
C_{3 T}=\frac{k_{\mathrm{B}} T \alpha_{0}}{16 \pi \varepsilon_{0}} \frac{\varepsilon(0)-1}{\varepsilon(0)+1} .
$$

For smaller distances $z \ll \hbar c /\left(k_{\mathrm{B}} T\right)$, the Matsubara sum is well approximated by an integral, so that

$$
\begin{aligned}
& U(z)=\frac{\hbar \mu_{0}}{16 \pi^{2}} \int_{0}^{\infty} \mathrm{d} \xi \xi^{2}\left[\alpha_{0}(\mathrm{i} \xi)+\alpha_{0}(-\mathrm{i} \xi)\right] \\
& \quad \times \int_{\xi / c}^{\infty} \mathrm{d} \kappa^{\perp} \mathrm{e}^{-2 \kappa^{\perp} z}\left[r_{s}-\left(2 \frac{\kappa^{\perp 2} c^{2}}{\xi^{2}}-1\right) r_{p}\right] .
\end{aligned}
$$

This distance region can be further divided into the retarded and nonretarded regimes. At retarded distances $c / \omega_{k 0} \ll z \ll \hbar c /\left(k_{\mathrm{B}} T\right)$, the approximations $\alpha_{0}(\mathrm{i} \xi) \simeq \alpha_{0}$ and $\varepsilon(\mathrm{i} \xi) \simeq \varepsilon(0) \equiv \varepsilon$ lead to

$$
U(z)=-\frac{C_{4}}{z^{4}}
$$

with

$$
\begin{aligned}
C_{4}=\frac{3 \hbar c \alpha_{0}}{64 \pi^{2} \varepsilon_{0}} \int_{1}^{\infty} \mathrm{d} v[ & \left(\frac{2}{v^{2}}-\frac{1}{v^{4}}\right) \frac{\varepsilon v-\sqrt{\varepsilon-1+v^{2}}}{\varepsilon v+\sqrt{\varepsilon-1+v^{2}}} \\
& \left.-\frac{1}{v^{4}} \frac{v-\sqrt{\varepsilon-1+v^{2}}}{v+\sqrt{\varepsilon-1+v^{2}}}\right]
\end{aligned}
$$

where we have introduced the new integration variable $v=\kappa^{\perp} c / \xi$. An explicit formula for $C_{4}$ and some of its limits are found in App. A. The values of $\alpha_{0}$ are $9.72 \times$ $10^{-39} \mathrm{C}^{2} \mathrm{~m}^{2} / \mathrm{J}$ and $1.19 \times 10^{-38} \mathrm{C}^{2} \mathrm{~m}^{2} / \mathrm{J}$ for $\mathrm{C}_{60}$ and $\mathrm{C}_{70}$, respectively. For nonretarded distances $z \ll c / \omega_{k 0}$, an asymptotic expansion in terms of $z$ leads to

$$
U(z)=-\frac{C_{3}}{z^{3}}
$$

with

$$
C_{3}=\frac{\hbar}{32 \pi^{2} \varepsilon_{0}} \int_{0}^{\infty} \mathrm{d} \xi\left[\alpha_{0}(\mathrm{i} \xi)+\alpha_{0}(-\mathrm{i} \xi)\right] \frac{\varepsilon(\mathrm{i} \xi)-1}{\varepsilon(\mathrm{i} \xi)+1} .
$$

Using the parameters of Sect. IIB, we have calculated the values of the coefficients $C_{3}, C_{4}$ and $C_{3 T}$ for $\mathrm{C}_{60}$ and $\mathrm{C}_{70}$ molecules interacting with a perfectly conducting surface as well as gold and silicon nitride surfaces. The results are given in Tab. VIII Due to its larger dipole moments, all coefficients are larger for $\mathrm{C}_{70}$ than they are for $\mathrm{C}_{60}$. The difference is most pronounced in the nonretarded regime. Comparing the coefficients for 


\begin{tabular}{|c||c|c|c|c|c|c|}
\hline \multicolumn{1}{|c||}{ Coefficient $\rightarrow$} & \multicolumn{2}{c|}{$C_{3}\left[\mathrm{Jm}^{3}\right]$} & \multicolumn{2}{c|}{$C_{4}\left[\mathrm{Jm}^{4}\right]$} & \multicolumn{2}{c|}{$C_{3 T}\left[\mathrm{Jm}^{3}\right]$} \\
\hline Material $\downarrow$ & $\mathrm{C}_{60}$ & $\mathrm{C}_{70}$ & $\mathrm{C}_{60}$ & $\mathrm{C}_{70}$ & $\mathrm{C}_{60}$ & $\mathrm{C}_{70}$ \\
\hline \hline Perfect conductor & $2.4 \times 10^{-47}$ & $3.0 \times 10^{-47}$ & $3.3 \times 10^{-55}$ & $4.0 \times 10^{-55}$ & $9.0 \times 10^{-50}$ & $1.1 \times 10^{-49}$ \\
$\mathrm{Au}$ & $1.0 \times 10^{-47}$ & $1.3 \times 10^{-47}$ & $3.3 \times 10^{-55}$ & $4.0 \times 10^{-55}$ & $9.0 \times 10^{-50}$ & $1.1 \times 10^{-49}$ \\
$\mathrm{Si}_{3} \mathrm{~N}_{4}$ & $8.4 \times 10^{-48}$ & $1.1 \times 10^{-47}$ & $1.5 \times 10^{-55}$ & $1.9 \times 10^{-55}$ & $5.5 \times 10^{-50}$ & $6.7 \times 10^{-50}$ \\
$\mathrm{SiN}_{x}$ & $6.3 \times 10^{-48}$ & $7.9 \times 10^{-48}$ & $1.4 \times 10^{-55}$ & $1.8 \times 10^{-55}$ & $5.3 \times 10^{-50}$ & $6.5 \times 10^{-50}$ \\
\hline
\end{tabular}

TABLE VIII: Coefficients for the asymptotic power laws of the CP potential of fullerene $(T=300 \mathrm{~K})$.

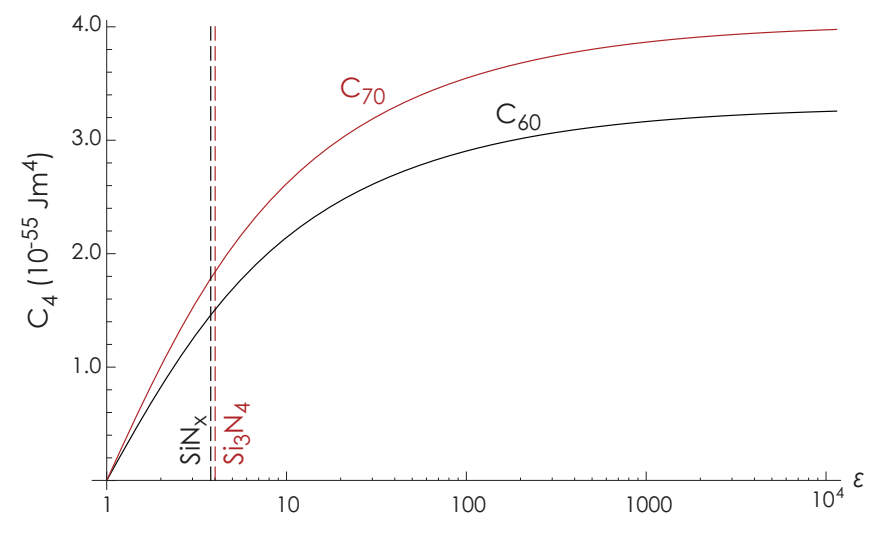

FIG. 4: Dependence of the retarded CP coefficient $C_{4}$ of $\mathrm{C}_{60}$ on the static permittivity.

the different materials, we note that Au can be considered a perfect metal in the retarded and thermal regimes, but corrections due to finite reflectivity are quite significant at nonretarded distances, leading to a reduction of $C_{3}$ by more than a factor of 2 . This is due to the fact that the molecular transition frequencies are a sizeable fraction of the Au plasma frequency. The silicon nitride potentials are smaller than those of $\mathrm{Au}$ due to their smaller permittivity. This difference is most pronounced at large distances. Comparing the two different silicon nitride species reveals that the coefficients for $\mathrm{Si}_{3} \mathrm{~N}_{4}$ are larger than those of $\mathrm{SiN}_{x}$ by up to $40 \%$ in the nonretarded regime.

The dependence of the retarded $\mathrm{CP}$ coefficient $C_{4}$ on the static permittivity of the surface material is displayed in Fig. 4. The figure shows that the close similarity of the $C_{4}$ coefficients for the two silicon nitride species is due to their similar static permittivities. It also reveals that the asymptotic $C_{4}$ value of a metal $(\varepsilon \rightarrow \infty)$ is only reached for very large permittivities, hence the large difference compared to Au.

The full potential of $\mathrm{C}_{60}$ in front of an $\mathrm{Au}$ surface has been calculated numerically and is displayed in Fig. 5 . The tabulated results can also be found in the supplementary materials. The figure shows that the potential is faithfully represented by the asymptotic power laws (3.2), (3.5) and (3.7) over a large part of the displayed distance range (as indicated by the shaded areas). However, there is a large gap between the nonretarded and retarded regions (between $10^{-7} \mathrm{~m}$ and $2 \times 10^{-8} \mathrm{~m}$ ) where neither limit applies. The potentials for $\mathrm{C}_{70}$ and for different surfaces show a similar qualitative behaviour.

The environment temperature $T$ affects the $\mathrm{CP}$ potential at distances larger than the thermal wavelength where thermal photons lead to softening of the potential decay. This temperature-dependence is demonstrated in Fig. 6] where we display the CP potential for different environment temperatures. As seen, thermal photons begin to affect the potential at smaller distances for higher environment temperatures, resulting in larger long-distance potentials. The potentials for $300 \mathrm{~K}$ and $600 \mathrm{~K}$ begin to differ from the zero-temperature result at distances larger than about $2 \mu \mathrm{m}$ or $4 \mu \mathrm{m}$, respectively.

In Fig. 7, we compare the potentials of the two different types of fullerenes. The $\mathrm{C}_{70}$ potential is larger than that of $\mathrm{C}_{60}$ by a factor which ranges between 1.3 at small distances and 1.2 at large distances. This difference is due to the larger dipole moments of $\mathrm{C}_{70}$, recall Eqs. (3.8) and (3.6) for the $C_{3}$ and $C_{4}$ coefficients and (2.5) for the atomic polarisability. The ratio of the potentials drops in the transition region between the nonretarded and retarded regimes. This is because the transition frequencies of $\mathrm{C}_{70}$ are slightly larger than those of $\mathrm{C}_{60}$.

The potentials of the two silicon nitride species are compared to that of $\mathrm{Au}$ in Fig. 8. Note that the curves for the two different fullerene molecules are indistinguishable in this plot. We see that the ratio is roughly $60 \%$ for both silicon nitride species at large distances. This similarity is due to their very similar static permittivities. At smaller distances, the potential of $\mathrm{Si}_{3} \mathrm{~N}_{4}$ is larger than that of $\mathrm{SiN}_{x}$ by $30 \%$, because its reflection coefficient falls off less rapidly with frequency.

As seen from Tables III and IV the line widths of the optical transitions are almost comparable to the transition frequencies. In our theory, the impact of the line widths is accounted for microscopically [26]: First, one explicitly solves the internal dynamics of the molecules, which depends on the transition frequencies and line widths. In a second step, this solution is used to determine the CP potential (3.1), where the dependence on the molecular parameters can be expressed via the molecular polarisability in a symmetrised form, $\frac{1}{2}\left[\alpha_{0}(\mathrm{i} \xi)+\alpha_{0}(-\mathrm{i} \xi)\right]$ [26]. As seen from Eq. (2.5), the line widths affect the imaginary-frequency polarisability most strongly at large frequencies. The largest impact on the CP potential 


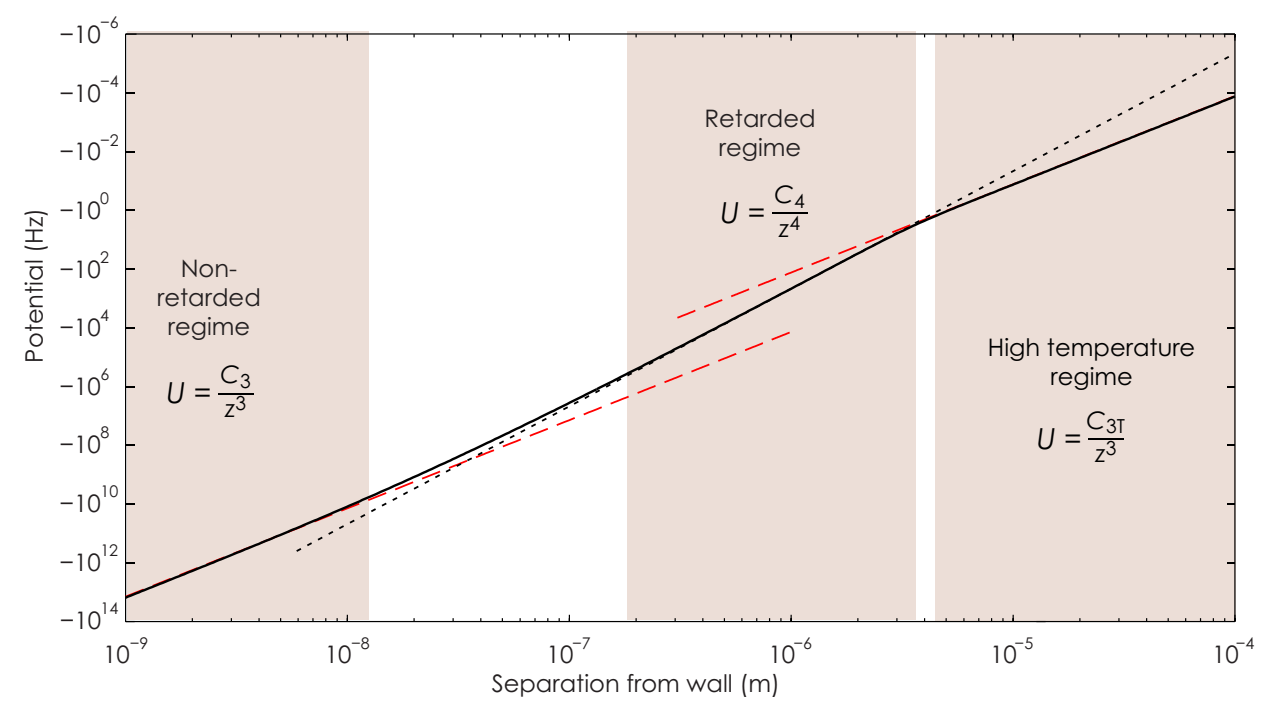

FIG. 5: $\mathrm{CP}$ potential of $\mathrm{C}_{60}$ in front of an Au surface at room temperature and its asymptotes.

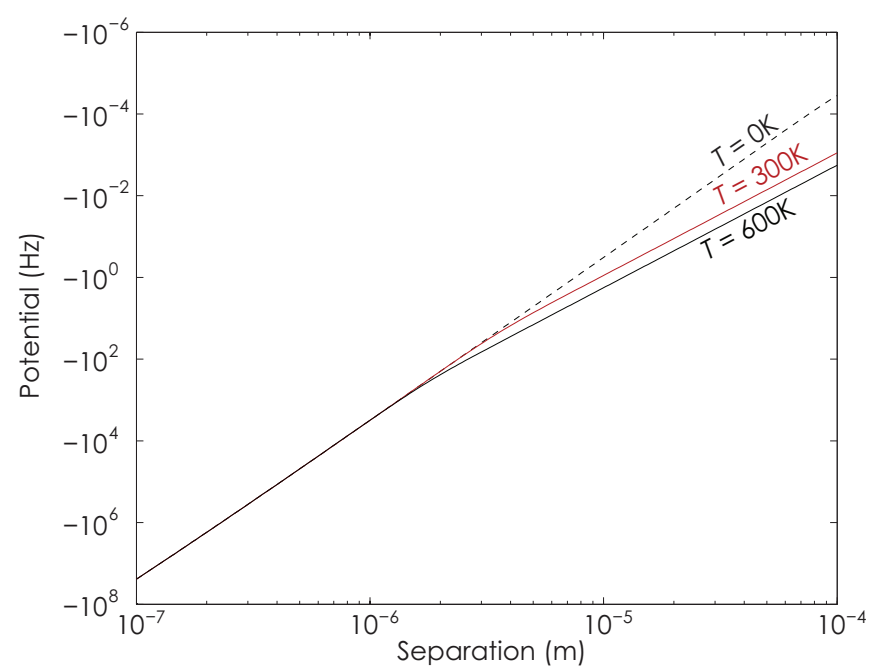

FIG. 6: $\mathrm{CP}$ potential of $\mathrm{C}_{60}$ in front of an $\mathrm{Au}$ surface at different temperatures.

is hence expected at small distances. In Table VIII we compare the $C_{3}$ coefficients including the finite line widths with those one would obtain for zero line widths. We note that the line widths have practically no influence on the nonretarded potential, with differences of about $1 \%$. This tiny change is quadratic in the line widths and unobservable in an experiment.

In an alternative approach based on linear-response theory [7], the atomic properties enter via the fluctuation-dissipation theorem. The resulting CP potential depends on the atomic polarisability in its unsymmetrised form,

$$
C_{3, \mathrm{LRT}}=\frac{\hbar}{16 \pi^{2} \varepsilon_{0}} \int_{0}^{\infty} \mathrm{d} \xi \alpha_{0}(\mathrm{i} \xi) \frac{\varepsilon(\mathrm{i} \xi)-1}{\varepsilon(\mathrm{i} \xi)+1} .
$$

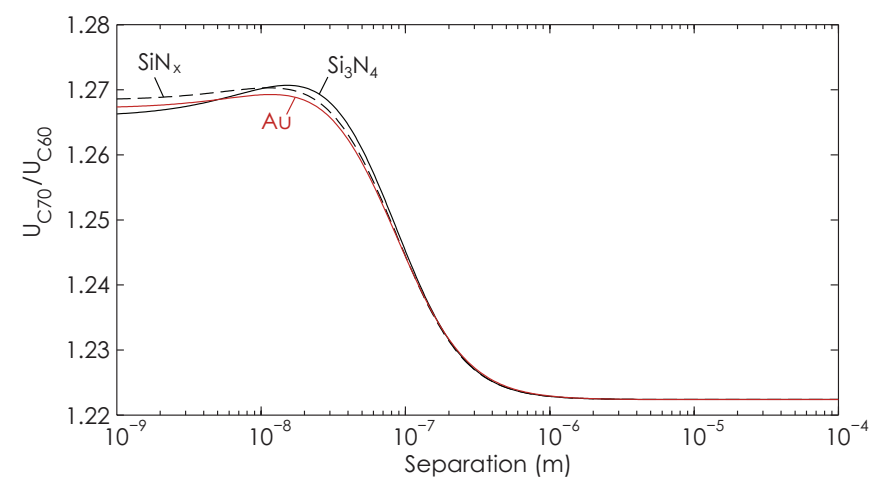

FIG. 7: Comparison of the $\mathrm{CP}$ potentials of $\mathrm{C}_{60}$ and $\mathrm{C}_{70}$ in front of gold and silicon nitride surfaces at room temperature.

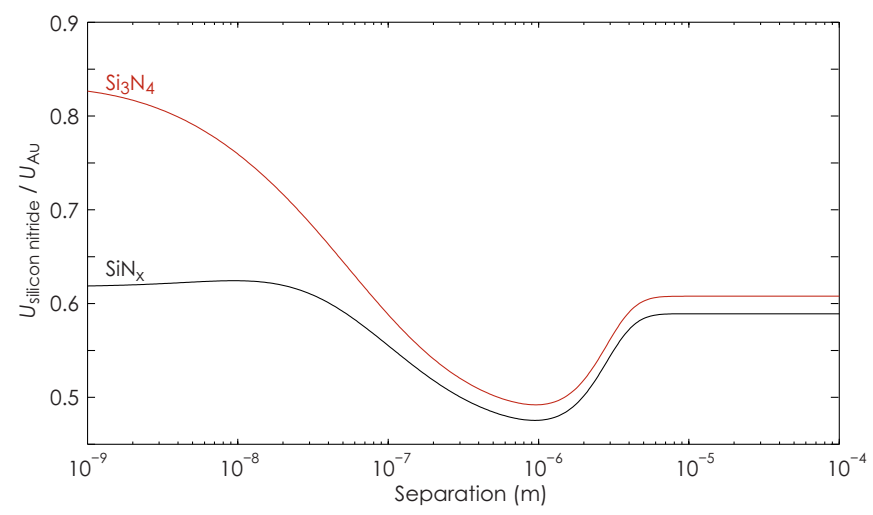

FIG. 8: Comparison of the CP potentials of fullerene in front of $\mathrm{Si}_{3} \mathrm{~N}_{4}$ and $\mathrm{SiN}_{x}$ surfaces at room temperature, normalised with respect to the corresponding Au potentials.

The atomic transition frequencies and line widths are not 


\begin{tabular}{|c||c|c|c|c|c|c|}
\hline \multicolumn{1}{|c||}{ Molecules $\rightarrow$} & \multicolumn{3}{|c|}{$\mathrm{C}_{60}$} & \multicolumn{3}{c|}{$\mathrm{C}_{70}$} \\
\hline Material $\downarrow$ & $C_{3}$ & $C_{3, \Gamma_{k} \rightarrow 0}$ & $C_{3, \mathrm{LRT}}$ & $C_{3}$ & $C_{3, \Gamma_{k} \rightarrow 0}$ & $C_{3, \mathrm{LRT}}$ \\
\hline \hline Perfect conductor & $2.36 \times 10^{-47}$ & $2.34 \times 10^{-47}$ & $2.15 \times 10^{-47}$ & $2.96 \times 10^{-47}$ & $2.93 \times 10^{-47}$ & $2.68 \times 10^{-47}$ \\
$\mathrm{Au}$ & $1.01 \times 10^{-47}$ & $1.00 \times 10^{-47}$ & $9.28 \times 10^{-48}$ & $1.27 \times 10^{-47}$ & $1.25 \times 10^{-47}$ & $1.15 \times 10^{-47}$ \\
$\mathrm{Si}_{3} \mathrm{~N}_{4}$ & $8.45 \times 10^{-48}$ & $8.36 \times 10^{-48}$ & $7.69 \times 10^{-48}$ & $1.06 \times 10^{-47}$ & $1.05 \times 10^{-47}$ & $9.55 \times 10^{-48}$ \\
$\mathrm{SiN}_{x}$ & $6.26 \times 10^{-48}$ & $6.21 \times 10^{-48}$ & $5.75 \times 10^{-48}$ & $7.86 \times 10^{-48}$ & $7.76 \times 10^{-48}$ & $7.11 \times 10^{-48}$ \\
\hline
\end{tabular}

TABLE IX: Impact of absorption on the $C_{3}$ coefficients $\left[\mathrm{Jm}^{3}\right]$ for the CP potential of fullerene.

considered explicitly, but only appear at the end of the calculation when specifying the polarisability. In particular, the line widths now affect the potential already to linear order. As seen from Table [X] this leads to a prediction of a reduction of the $C_{3}$ coefficient by about $10 \%$ due to absorption. This effect could be visible in sufficiently accurate $C_{3}$ experiments, making it possible to distinguish between the macroscopic, linear response model for absorption and our microscopic model (which predicts that the effect of absorption on ground-state potentials is negligible). This possibility makes fullerenes most attractive for CP-potential studies. In contrast, atomic systems are unable to resolve the difference between the effects of symmetrised vs unsymmetrised polarisabilities (or the neglect of line widths altogether).

Finally, let us discuss the impact of the infrared resonances on the $\mathrm{C}_{60}$ potential. As seen from Table VI, their dipole moments are much smaller than those of the optical transitions (Table III). On the other hand, their transition wavelengths being much longer than those of the optical transitions, the nonretarded limit applies over a larger range of distances. In addition, the thermal photon numbers can take large values even at room temperature, so that resonant potentials (2.7) come into play.

At distances up to about $10 \mu \mathrm{m}$, the $\mathrm{CP}$ potential due to phonon resonances is strongly nonretarded. As shown in Ref. [27], the potential in this regime is well approximated by

$$
U_{\text {Phonon }}(z)=-\frac{C_{3, \text { Phonon }}}{z^{3}}
$$

with

$$
C_{3, \text { Phonon }}=\frac{1}{48 \pi \varepsilon_{0}} \sum_{n, k} p_{n}\left(T_{\mathrm{m}}\right)\left|\boldsymbol{d}_{n k}\right|^{2}
$$

where the sum only runs over phonon transitions. This result holds regardless of the environment temperature for all materials of sufficiently large permittivity. The corresponding $C_{3}$ coefficient depends on the internal temperature of the molecule, it ranges from $C_{3, \text { Phonon }}=$ $3.4 \times 10^{-51} \mathrm{Jm}^{3}$ at zero temperature to $C_{3, \text { Phonon }}=2.6 \times$ $10^{-51} \mathrm{Jm}^{3}$ at $T_{\mathrm{m}}=300 \mathrm{~K}$. A comparison with the $C_{3}$ coefficients listed in Table VIII reveals that the potential contribution from infrared phonon transitions is smaller than the discussed potential from optical transitions by more than two orders of magnitude.
At larger distances, corrections due to imperfect reflectivity manifest themselves [28]. However, they do not affect the order of magnitude of the phonon CP potential, which remains insignificant. As we have numerically verified, the phonon contributions only become relevant at very large distances, well beyond $100 \mu \mathrm{m}$.

\section{SUMMARY}

We have determined the Casimir-Polder interaction of $\mathrm{C}_{60}$ and $\mathrm{C}_{70}$ with plane surfaces of $\mathrm{Au}$ and two different nitride species, as commonly used in molecular interference experiments. The numerically calculated potentials is well approximated by the thermal, retarded and nonretarded asymptotes for large, intermediate and small distances, respectively. We have found that the potential is entirely due to optical transitions and hence independent of the internal temperature of the molecules. The environment temperature affects the potential for distances larger than $2 \mu \mathrm{m}$ at room temperature. Comparing the potentials for different silicon nitride species, we have found differences of up to $30 \%$ in the nonretarded regime, which is most relevant for diffraction experiments.

According to our microscopic theory of the moleculefield interaction, the finite line widths of the molecules have practically no influence on the Casimir-Polder potential. A macroscopic linear-response approach, on the other hand, predicts that they decrease the nonretarded potential by about $10 \%$. The relatively large line widths of the fullerene transitions thus make them an ideal system to study the impact of molecular absorption on dispersion interactions.

\section{Acknowledgments}

We thank H. Ulbricht and S. Nimmrichter for discussions. Support from the European Science Foundation (ESF) within the activity 'New Trends and Applications of the Casimir Effect' is gratefully acknowledged. A. J. and K. H. acknowledge support by the MIME project within the ESF Eurocore EuroQUASAR program. 


\section{Appendix A: Coefficient for the retarded Casimir-Polder potential}

The integral of the coefficient $C_{4}$, given in Eq. (3.6) can be solved explicitly with the result

$$
\begin{aligned}
C_{4}= & \frac{3 \hbar c \alpha_{0}}{64 \pi^{2} \varepsilon_{0}}\left\{\frac{10-3 \sqrt{\varepsilon}-4 \varepsilon-3 \varepsilon^{3 / 2}+6 \varepsilon^{2}}{3(\varepsilon-1)}\right. \\
& -\frac{\varepsilon^{2}}{\sqrt{\varepsilon+1}}\left[\log \frac{\sqrt{\varepsilon+1}-1}{\sqrt{\varepsilon+1}+1}+2 \log (\sqrt{\varepsilon}+\sqrt{\varepsilon+1})\right] \\
& \left.-\frac{2 \varepsilon^{3}-4 \varepsilon^{2}+3 \varepsilon+1}{(\varepsilon-1)^{3 / 2}} \log (\sqrt{\varepsilon}+\sqrt{\varepsilon-1})\right\}
\end{aligned}
$$

For large and small values of $\varepsilon, C_{4}$ behaves like

$$
\begin{aligned}
& C_{4} \sim \frac{3 \hbar c \alpha_{0}}{64 \pi^{2} \varepsilon_{0}}\left(2-\frac{5}{2 \sqrt{\varepsilon}}+\frac{44}{15 \varepsilon}+\ldots\right), \quad \varepsilon \gg 1 \\
& C_{4} \sim \frac{3 \hbar c \alpha_{0}}{64 \pi^{2} \varepsilon_{0}}\left(\frac{23}{30} \chi-\frac{169}{420} \chi^{2}+\ldots\right), \quad \chi \ll 1
\end{aligned}
$$

[1] M. Arndt, O. Nairz, J. Voss-Andreae, C. Keller, G. van der Zouw and A. Zeilinger, Nature 401, 680 (1999).

[2] K. Hornberger, S. Gerlich, P. Haslinger, S. Nimmrichter and M. Arndt, Rev. Mod. Phys. 84, 157 (2012) .

[3] R. E. Grisenti, W. Schöllkopf, J. P. Toennies, G. C. Hegerfeldt, and T. Köhler, Phys. Rev. Lett. 83, 1755 (1999).

[4] S. Nimmrichter and K. Hornberger, Phys. Rev. A 78, 023612 (2008).

[5] S. Gerlich et al., Nat. Phys. 3, 711 (2007).

[6] S. Scheel and S. Y. Buhmann, Acta Phys. Slovaca 58, 675 (2008).

[7] C. Henkel, K. Joulain, J.-P. Mulet and J.-J. Greffet, J. Opt. A: Pure Appl. Opt. 4, S109 (2002).

[8] M.-P. Gorza and M. Ducloy, Eur. Phys. J. D 40, 343 (2006).

[9] S. Y. Buhmann and S. Scheel, Phys. Rev. Lett. 100, 253201 (2008).

[10] M. Bordag, B. Geyer, G. L. Klimchitskaya and V. M. Mostepanenko, Phys. Rev. B 74, 205431 (2006).

[11] S. Å. Ellingsen, S. Y. Buhmann and S. Scheel, Phys. Rev. A 79, 052903 (2009).

[12] E. Sohmen, J. Fink and W. Krätschmer, Z. Phys. B 86, 87 (1992).

[13] N. J. Ju, A. Bulgac, J. W. Keller, Phys. Rev. B 48, 9071 (1993).

[14] P. C. Eklund, A. M. Rao, Y. Wang, P. Zhou, K.-A. Wang, J. M. Holden, M. S. Dresselhaus, G. Dresselhaus, Thin Solid Films 257, 211 (1995).
[15] A. Lambrecht, S. Reynaud, Eur. Phys. J. D 8, 309 (2000).

[16] R. S. Decca, D. Lopez, E. Fischbach, G. L. Klimchitskaya, D. E. Krause, V. M. Mostepanenko, Eur. Phys. J. C 51, 963 (2007).

[17] V. A. Parsegian and G. H. Weiss, J. Colloid Interf. Sci. 81, 285 (1981).

[18] E. D. Palik (ed.), Handbook of Optical Constants of Solids (New York: Academic Press, 1995).

[19] R. Brühl, P. Fouquet, R. E. Grisenti, J. P. Toennies, G. C. Hegerfeldt, T. Köhler, M. Stoll and C. Walter, Europhys. Lett. 59, 357 (2002).

[20] E. D. Palik (ed.), Handbook of Optical Constants of Solids II (Academic Press, New York, 1991).

[21] F. Gervais and B. Piriou, J. Phys. C - Solid St. Phys. 7, 2374 (1974).

[22] R. Mitzner and E. E. B. Campbell, J. Chem. Phys. 103, 2445 (1995).

[23] L. Hackermüller, K. Hornberger, B. Brezger, A. Zeilinger and M. Arndt, Nature 427, 711-714 (2004).

[24] K. Hornberger, L. Hackermüller and M. Arndt, Phys. Rev. A 71, 023601 (2005).

[25] E. M. Lifshitz, Sov. Phys. JETP 2, 73 (1956).

[26] S. Y. Buhmann, L. Knöll, D.-G. Welsch and D. T. Ho, Phys. Rev. A 70, 052117 (2004).

[27] S. Å. Ellingsen, S. Y. Buhmann and S. Scheel, Phys. Rev. Lett. 104, 223003 (2010).

[28] S. Å. Ellingsen, S. Y. Buhmann and S. Scheel, Phys. Rev. A 84, 060501(R) (2011). 